\title{
Reducir tiempos de espera de pacientes en el departamento de emergencias de un hospital utilizando simulación
}

${ }^{(1)}$ Silvia V. Medina León
${ }^{(2)}$ Amalia Medina Palomera
${ }^{(3)}$ Álvaro González Ángeles

\section{INTRODUCCIÓN}

\section{RESUMEN}

Se desarrolló un modelo de simulación discreta del departamento de Obstetricia-Ginecología de emergencias de un hospital público de la región. Se utilizó el software Arena de Rockwell Automation para analizar el flujo de pacientes, identificar los cuellos de botella y proponer mejoras que reduzcan los tiempos de espera de los pacientes durante su estancia. El modelo de simulación cubrió desde la llegada del paciente al módulo de información hasta la salida del área de recuperación. Se utilizó la evaluación de escenarios para determinar las propuestas de mejora obteniéndose una reducción de un $76 \%$ en tiempos de espera total.

Palabras clave: Simulación, simulación de eventos discretos, tiempos de espera.

REDUCE WAITING TIMES IN A HOSPITAL EMERGENCY DEPARTMENT USING SIMULATION

\section{ABSTRACT \\ A discrete simulation model was developed for the obstetrics-gynecology emergency department of a regional public hospital. It was utilized the software Arena of Rockwell Automation to analyze the flow of patients, identify bottlenecks and propose im- provements that reduce patient waiting times. The simulation model included from the patient arrival at the information module until the patient leaves the recuperation area in emergency. Scenarios evaluation was utilized to determine improvement proposals giving as result a $76 \%$ reduction of total waiting times.}

Key words: Simulation, discrete event simulation, waiting times. et al., 2005).
La simulación, tal como lo expresa Kelton (Kelton, 2004) es el proceso de diseñar y crear un modelo computarizado de un sistema real o propuesto con el propósito de conducir experimentos numéricos para entender el comportamiento del sistema dadas ciertas condiciones. Se le agregan los términos de simulación de eventos discretos cuando las variables de estado no cambian de manera continua, sino, mas bien, en instantes de tiempo no periódicos en la ocurrencia de eventos (Guasch

Simulación es una de las herramientas recomendadas en la solución de problemas de sistemas complejos donde la utilización de modelos matemáticos no es práctica (Taha, 1995). En el sector de servicios es una herramienta utilizada frecuentemente y los hospitales y clínicas están dentro de este caso. El sistema de un hospital tiene muchas variables y diferentes eventos aleatorios por lo que en la mayoría de los planteamientos de problemas se consideran sistemas complejos. Diferentes artículos han publicado aplicaciones de simulación en el sector salud, entre ellos está Clague (Clague et al., 1997), quien utilizó la simulación para proponer soluciones en la reducción de tiempos de espera de pacientes para consulta en una clínica. Akkerman (Akkerman, 2004) utilizó la simulación para la asignación de camas a las salas de cirugía cardiaca para reducir tiempos de espera. Denton (Denton y cols., 2007) utilizó la simulación para la secuenciación óptima de cirugías. Lapierre (Lapierre, 2007) utilizó la simulación para planear las actividades de logística de cuándo comprar un producto, entregarlo a los diferentes departamentos, cuándo un empleado debe de trabajar y qué tareas debe de hacer. En cada uno de los trabajos, se presenta un enfoque de optimización diferente pero todos tienen el objetivo de ofrecer un mejor servicio optimizando los recursos.

En el caso bajo estudio, la problemática que se presenta es reducir los tiempos de espera de pacientes del área de obstetricia-ginecología, abarcando desde su llegada hasta la salida del área de emergencias. Se desarrolló un modelo del sistema real a través de la simulación utilizando el software Arena de Rockwell Automation, donde se pudieron identificar los cuellos de botella principales. Se llevó a cabo un análisis de escenarios para determinar una propuesta de mejora que redujera los tiempos de espera de forma significativa.

Estudiante de doctorado en Ingeniería Industrial; Profesora de la Facultad de Ingeniería de la Universidad Autónoma del Estado de Baja California, México. E-mail: vmedina@uabc.mx / vmedina88@yahoo.com

2 Doctora en ingeniería; Profesora investigadora del Instituto Tecnológico de Mexicali, México. E-mail: amedinapalomera@yahoo.com.mx

3 Doctor en ciencias; Profesor investigador de la Facultad de Ingeniería de la Universidad Autónoma del Estado de Baja California, México. E-mail: gangelesa@uabc.mx / gangelesa@yahoo.com.mx 


\section{METODOLOGÍA}

La metodología utilizada está basada en el método propuesto por Guash (Guash et al. 2005). A continuación, se describe el desarrollo de los pasos:

\section{Formulación del problema}

Esta etapa se llevó a cabo a través de reuniones con personal del hospital y visitas al área. El objetivo del proyecto se definió en reducir los tiempos de espera de los pacientes al menos en 30\% durante su estancia en el área de obstetricia-ginecología del departamento de emergencias. El personal del hospital enfatizó su interés especial en analizar los recursos de camas en el área de ObstetriciaGinecología del departamento de emergencias y, además, solicitó que la propuesta de mejora no incluyera modificaciones en la planta de doctores y enfermeras.

\section{Diseño del modelo conceptual}

En esta etapa, se desarrollaron diagramas de flujo detallados para el área de Obstetricia-Ginecología del departamento de emergencias. Para efectos de simplificación, en la figura 1 , se presenta un diagra- ma de flujo general del proceso de atención de pacientes. La descripción del sistema se divide en 5 áreas físicas, siendo estas: recepción, revisión, observación, salas de expulsión y quirófano, y recuperación. La descripción general del proceso se presenta a continuación:

- Recepción. De manera general, las pacientes llegan a información, pasan al mostrador si están afiliadas al servicio; y si no, pasan a caja; después, pasan a la sala de espera especial para las pacientes de obstetricia-ginecología. Ahí, esperan hasta que el doctor de revisión esté disponible y las pase al área de revisión.

- Revisión. El área de revisión cuenta con dos camas y está a cargo de un doctor. El doctor lleva a cabo la revisión de la paciente y determina si requiere hospitalización o no. Si no requiere hospitalización, termina la consulta y la paciente se retira. En caso de que requiera hospitalización, la paciente pasa a la sala de observación.

- Observación. La sala de observación cuenta con 7 camas y es donde se prepara a la paciente para su estancia y donde permanece para ser monitoreada hasta que se lleve a cabo el procedimiento correspondiente. En caso que no haya cama disponible, la preparación de la paciente

Figura 1: Diagrama de flujo general del proceso de atención de pacientes
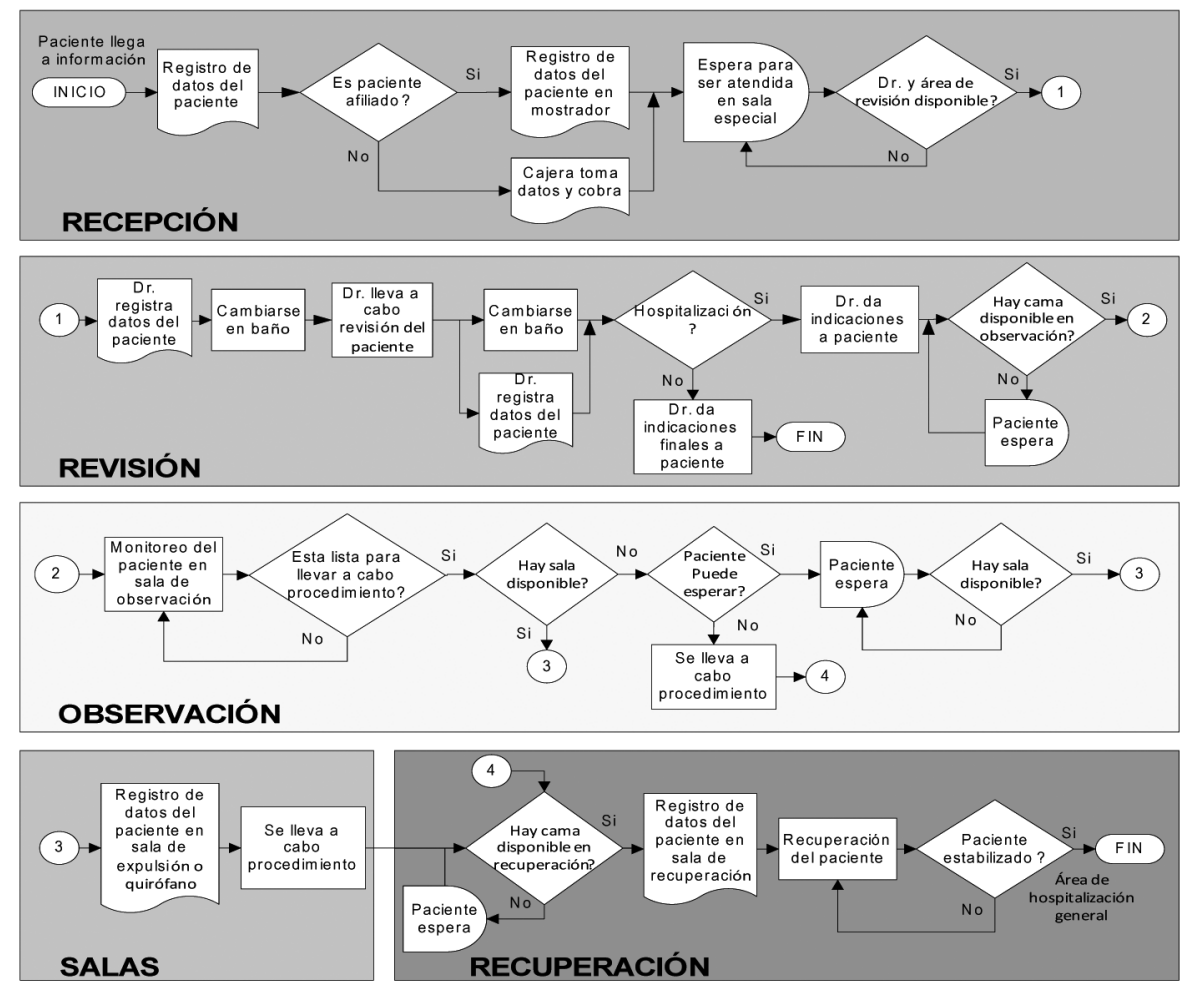

Fuente: Datos observados en el área de Obstetricia-Ginecología del departamento de emergencias de un hospital público de la región. 
se efectúa en el área de revisión. En teoría, para las pacientes embarazadas, se considera el ingreso a la sala de observación cuando su estancia esperada es menor a 12 horas, pero en la práctica, este dato es mayor. Cuando la paciente está lista para que se realice el procedimiento, se transporta a una de las salas de expulsión o quirófano.

- Salas de expulsión y quirófano. Hay tres salas de expulsión y dos de quirófanos; los quirófanos son utilizadas para los procedimientos quirúrgicos como cesáreas y las salas de expulsión son utilizadas para procedimientos como partos normales y otros procedimientos que no involucran una cirugía mayor. Los procedimientos que se llevan a cabo son: salpingo, biopsia cérvix, electrofulguración, electrocirugía, crioterapia, biopsia endometrio, biopsia mama, parto normal, cesárea, legrado, laparotomía exploratoria y colpoperineoplastía. Aunque sucede esporádicamente, en los casos de parto en que no hay ninguna sala disponible, el procedimiento se lleva a cabo en la sala de observación. Una vez efectuado el procedimiento correspondiente, el paciente es trasladado a la sala de recuperación.

- Recuperación. La sala de recuperación cuenta con 13 camas y es donde el paciente permanece por un par de horas para monitorear su estado. Luego de que el paciente se establece, este abandona el área de emergencias y se traslada al área de hospitalización general, donde terminará su recuperación para posteriormente abandonar el hospital.

\section{Colección y tratamiento de los datos}

En esta etapa, se llevaron a cabo las siguientes actividades:

3.1 Identificar información requerida. En base al estudio inicial del proceso se preparó una lista de los datos requeridos. De manera resumida, los datos requeridos fueron: llegada de pacientes, tiempos de procesos, registros de hora de inicio de las actividades a lo largo del sistema, porcentajes de ocurrencias de procesos en nodos de decisión, recursos requeridos y disponibles de camas, salas, enfermeras y doctores.

3.2 Colección de información. De la lista de datos requeridos, se investigó qué datos estaban disponibles en registros históricos y qué datos se tenían que obtener por muestreo directamente del sistema. Para los datos que se requerían muestrear, se prepararon formatos y se obtuvie- ron muestras iniciales de 30 observaciones. En base a la muestra obtenida, se obtuvo el error utilizando la ecuación 1.

$$
E=t_{n-1,1-\frac{\alpha}{2}} \frac{S}{\sqrt{n}} E=t_{n-1,1-\frac{\alpha}{2}} \frac{S}{\sqrt{n}}
$$

Ec. 1

Donde $E$ es el error de la muestra, $S$ es la desviación estándar de la muestra, t es el valor de tablas t-student, $\mathrm{n}$ es el tamaño de la muestra y $\alpha$ es el nivel de significancia del 5\%. En los casos en los que el error excedía un $5 \%$ de la media, se incrementaba el tamaño de la muestra tomando como aproximación la ecuación 2.

$$
n \cong Z_{1-\frac{\alpha}{2}}^{2} \frac{S^{2}}{E^{2}} n \cong Z_{1-\frac{\alpha}{2}}^{2} \frac{S^{2}}{E^{2}}
$$

La ecuación 2 es igual que la uno, solo que se sustituye el valor de la distribución t-student por el valor $Z$ de la distribución normal debido a que el tamaño de la muestra será más de 30. Otra observación en la ecuación es que se utiliza la $S$ obtenida de la muestra inicial considerando que es la misma que el de una muestra más grande (Kelton, 2004). Obtenido el tamaño de muestra requerido, se concluyó con la colección de datos.

3.3 Preparación de datos en Excel. Los datos colectados se introdujeron en hojas en Excel, se obtuvieron sus datos como media, valor mínimo, valor máximo, desviación estándar y error para su posterior utilización en la validación.

3.4 Ajuste de datos a una distribución de probabilidad. Cada uno de los datos muestreados de entrada se ajustaron a una distribución de probabilidad utilizando la herramienta "Analizador de datos de entrada" (Input Analyzer). El analizador de datos de entrada presenta el error de ajuste de los datos para cada una de las distribuciones, así como los resultados de la prueba Chi-cuadrada $\left(\mathrm{X}^{2}\right)$ para determinar la discrepancia entre la distribución observada y la teórica.

De forma particular, las llegadas de los pacientes o arribos se ajustaron a una distribución exponencial no estacionaria debido a que la razón de arribos varía sobre el tiempo (Law y Kelton, 2000). En arena esta función se incorporó al utilizar la opción "Plan" (Schedule) obteniendo 
Figura 2: Vista de la ventana para definir patrón de tiempo de llegadas.

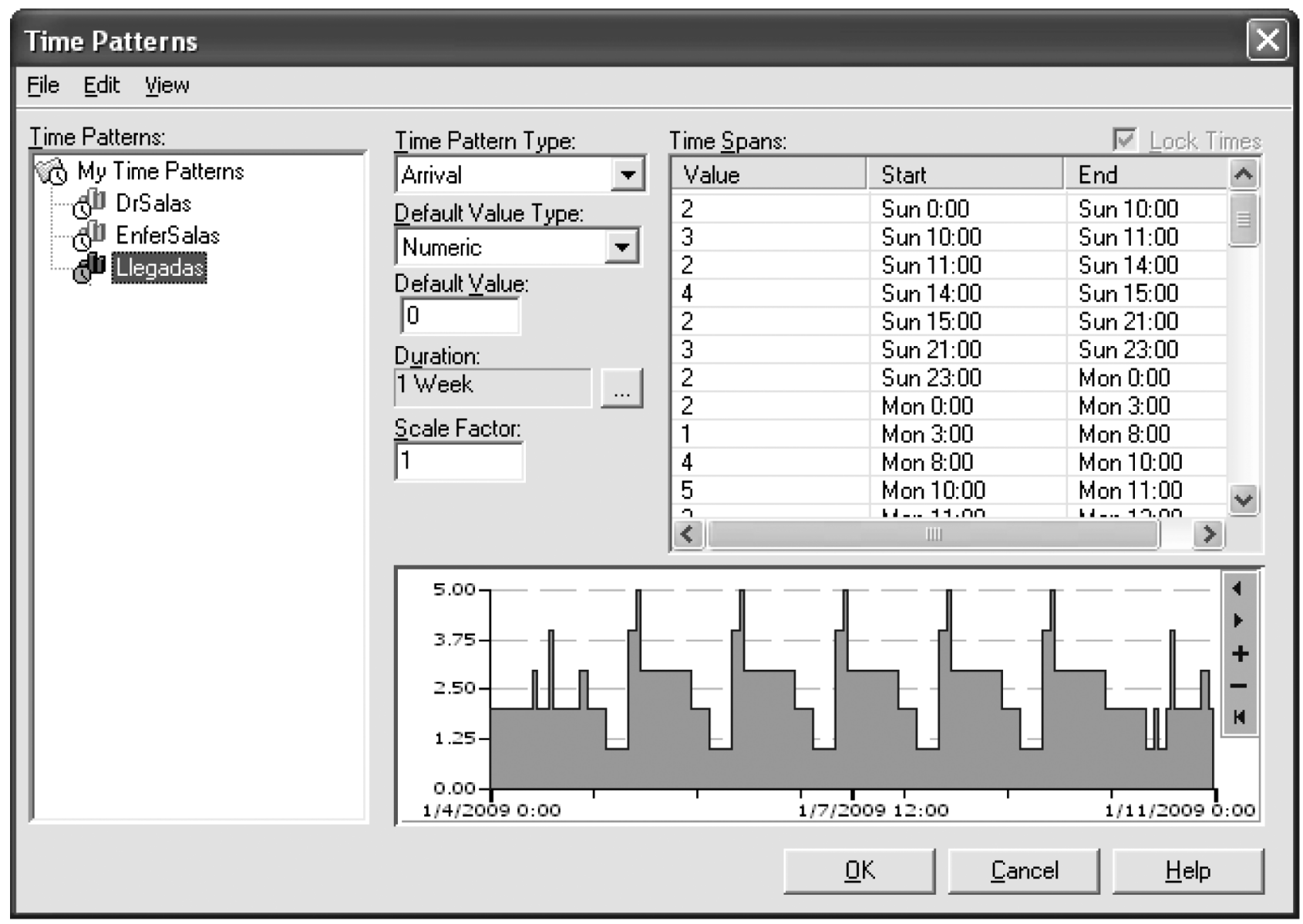

Fuente: Datos observados en el área de Obstetricia-Ginecología del departamento de emergencias de un hospital público de la región.

previamente promedios de arribos por hora. La figura 2 muestra la ventana para la entrada de datos de la razón de arribos por hora.

cabe hacer mención que los procesos que solo tenían valores de tiempo aproximado, debido a que la ocurrencia de los eventos era escasas, se ajustaron a la distribuciones triangulares o uniformes (Law y Kelton,2000).

\section{Construcción del modelo}

La construcción del modelo se divide en la programación de la lógica, el diseño de la animación del modelo y especificar el tiempo de estabilización del modelo. A continuación, se describe cada uno de estos puntos.

4.1 Programación de la lógica del modelo. La programación de la lógica del modelo consistió en el desarrollo de 9 sub-modelos, los cuales se muestran en la figura 3.

Figura 3: Sub-modelos de la construcción del modelo

La programación de arena es a través de bloques semejando diagramas de flujo junto con información de tablas y funciones complementarias. La figura 4 muestra como ejemplo la programación dentro del sub-modelo 1 denominado "llegadas".

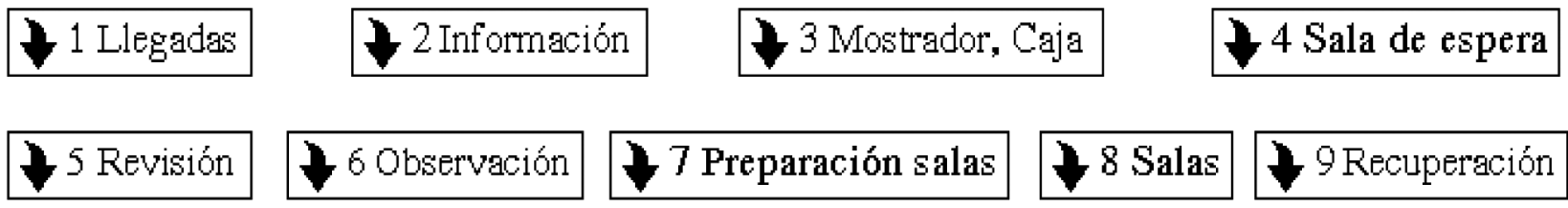


Figura 4: Programación dentro del sub-modelo 1 denominado "llegadas"

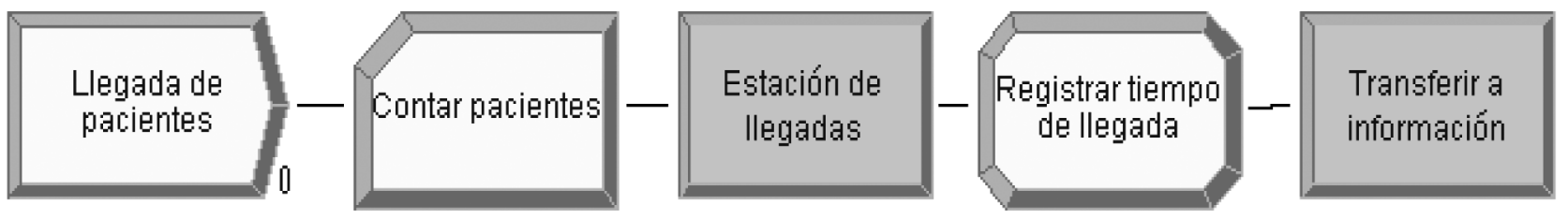

4.2 Diseño de la animación del modelo. La animación del modelo consistió en el diseño de la distribución actual incorporando recursos claves como camas y personal. Los aspectos sobresalientes de la animación permiten ver el acumulamiento de las personas y sus movimientos entre áreas; con respecto a los recursos, estos cambian de color cuando están siendo utilizados. Además de la distribución, la animación presenta dos secciones más denominadas: variables y gráficas. Ambas secciones presentan información en tiempo de simulación real, ya sea numérica o gráfica, de manera respectiva sobre datos de interés como número de personas esperando, tiempos de espera promedio y porcentajes de utilización de los recursos. La figura 5 muestra la vista de la animación del modelo.

4.3 Especificar el tiempo de estabilización del modelo (Warm-up). A diferencia de un sistema de simulación terminal, como el de una tienda que abre a las 9:00 a.m. sin clientes y cierra a las 8:00 p.m. nuevamente sin clientes, el sistema de emergencias de un hospital es de estado estacionario donde todo el día hay pacientes dispersos en el sistema. Debido a lo anterior, se requiere especificar un tiempo de estabilización del modelo (Kelton, 2004). Para determinar el tiempo de estabilización, se seleccionó un parámetro característico del modelo, en este caso fue el número de pacientes en el sistema. Se hicieron 10 corridas de 360 horas cada una. La gráfica obtenida por la herramienta "Analizador de salidas" (Output Analyzer) se muestra en la figura 6 , donde se aprecia que el sistema se estabiliza a las 40 horas, con la finalidad de redondear el valor en días, el tiempo de estabilización se estableció en dos días o 48 horas. Otros parámetros que pudieran representar la estabilidad del sistema fueron de igual forma analizados como es el caso del número de salas utilizadas, número de pacientes en el área de observación y de recuperación, pero su gráfica se estabilizaba en 24 horas; debido a que el valor es menor, se optó solo por presentar la gráfica del número de pacientes total en el sistema.

\section{Verificación}

La verificación consiste en revisar que el modelo se comporte como el programador espera (Kelton, 2004). Esta tarea se llevó a cabo a la par de la programación. Las técnicas de verificación utilizadas fueron:

5.1 Reuniones con responsables del proceso. Las reuniones se llevaron a cabo para presentar la forma en que el programador visualizaba las

Figura 5: Vista de la animación del modelo

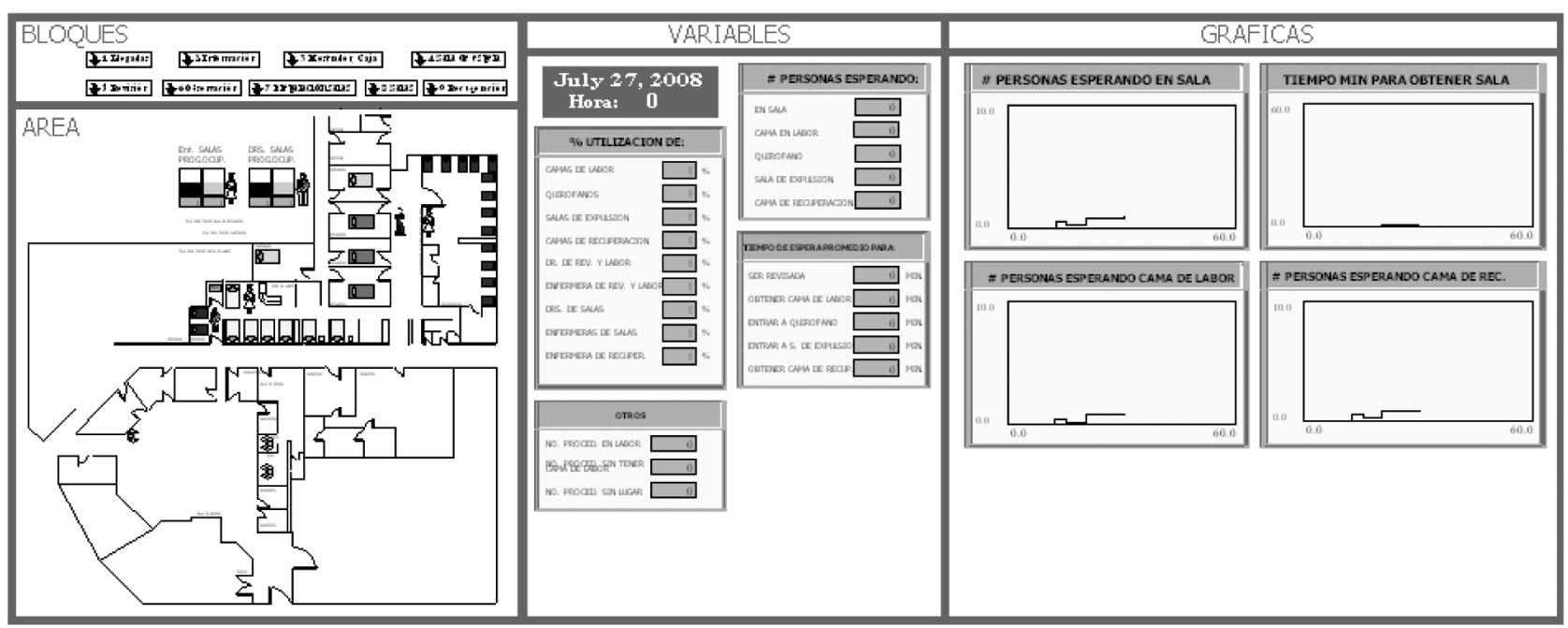


Figura 6: Gráfica del número de pacientes en el sistema utilizada para determinar el tiempo de estabilización del sistema.

Número de pacientes en el sistema

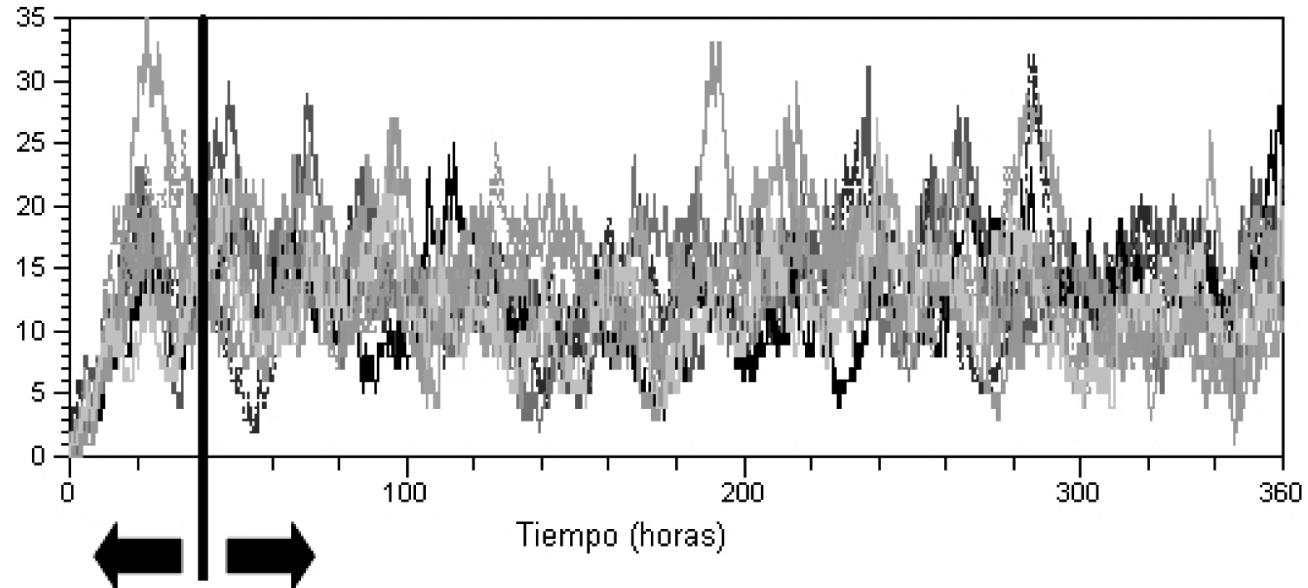

Sistema inestable Sistema estable

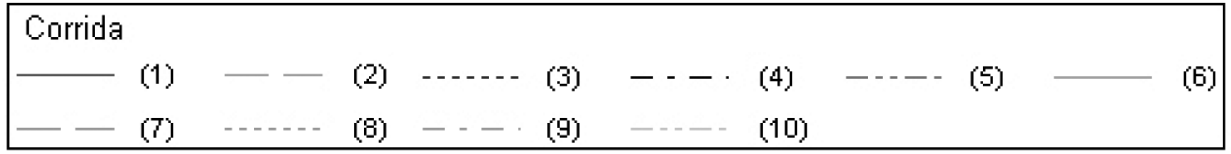

Fuente: Gráfica generada por el modelo de simulación.

operaciones del área con la finalidad de que coincidiera con la perspectiva de los responsables de las áreas.

5.2 Animación. La animación fue una herramienta de verificación tanto para el programador como los responsables del proceso al permitir visualizar que las operaciones funcionaran como se esperaba.

\subsection{Herramienta "Controlador de corrida" (Run con-} troller) de Arena. La herramienta Controlador de corrida permite, entre otras cosas, ejecutar el modelo enfocándose en una entidad, proceso específico o variable visualizando el código fuente de Arena denominado SIMAN. Esto se logra a través de comandos específicos en lenguaje SIMAN. De esta manera, el programador visualizó la secuencia de la ejecución de las operaciones y llevó a cabo las correcciones debidas a malinterpretaciones sobre la secuencia de operaciones. La figura 7 muestra la utilización del comando "Step" ("paso") en la ventana del Controlador de corrida.

Figura 7: Vista del Controlador de corrida

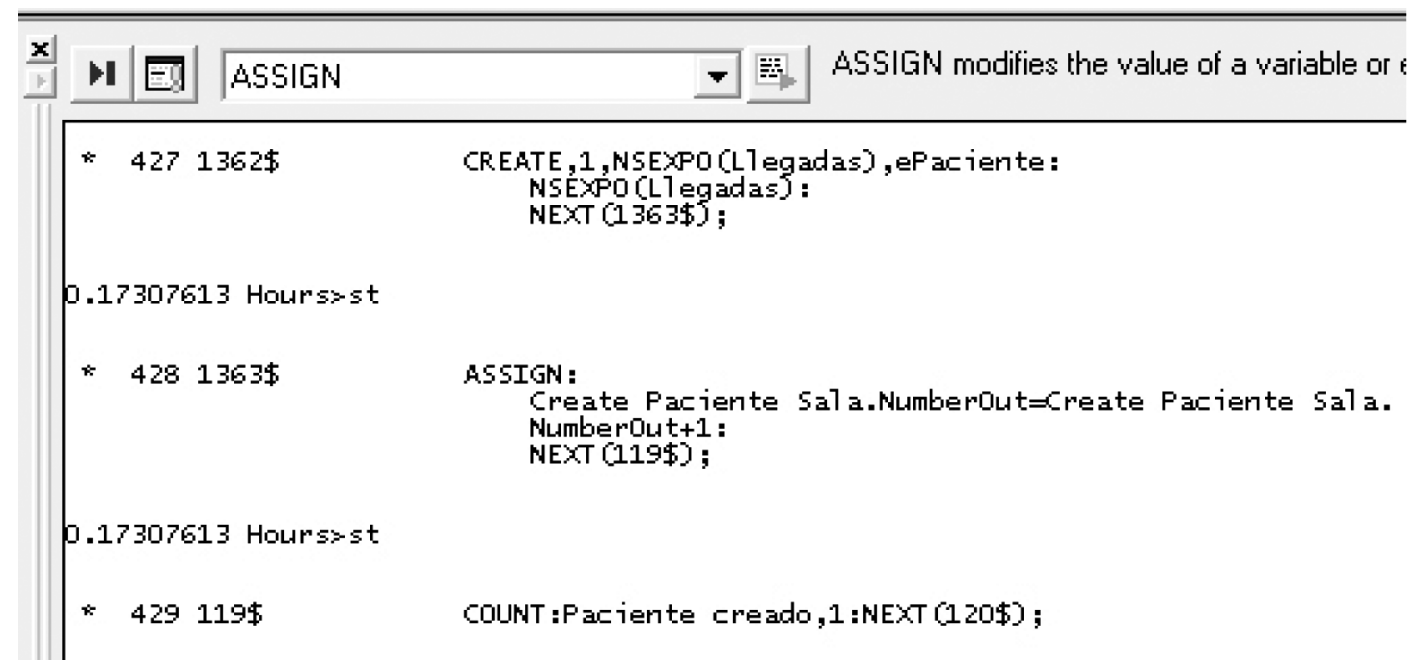


5.4 Reportes de simulación. Los reportes de simulación se consultaron constantemente y se comparaban con los datos muestreados de manera que fueran valores razonables. Durante la verificación, se hacía una sola corrida larga con una duración de 930 días, es decir, una muestra de 30 meses de 31 días debido a que el mes simulado fue diciembre.

\section{Validación}

La validación se refiere a asegurarse que el modelo se comporte como el sistema real (Kelton, 2004). En la validación, se compararon los resultados del modelo de 10 corridas de 930 días de duración con los del sistema real. En base a los resultados aceptables de los errores obtenidos (Half widhts), no se requirió hacer más corridas. A continuación, se presentan los resultados validados característicos del modelo.

\section{Análisis y experimentación}

En primer lugar, se inició con un análisis de tiempos de espera de los pacientes en las filas. Este análisis consiste en identificar las filas con mayor tiempo de espera de los pacientes. La tabla 3 presenta los tiempos promedios de espera de los pacientes ordenados de forma descendente. Se observa que tomar cama en observación es la fila que tiene el tiempo promedio de espera más alto seguido por tomar lugar en revisión. Estos dos tiempos de espera, además de ser los más altos, también son de los más críticos, porque se refieren a los tiempos de espera de los pacientes para ingresar al área de observación y ser atendidas, respectivamente. La suma de los tiempos de espera promedio resulta en 0.67066 horas. También se puede observar en tabla 3 el número de personas esperando en las respectivas filas, mostrando de igual manera los valores más altos para las filas de tomar cama en observación y tomar lugar en revisión.

La experimentación consistió en utilizar escenarios modificando el número de camas adicionales en 2 , 4 y 6 , es decir, 9,11 y 13 camas totales en el área de observación. Como referencia, observe la tabla 3 que el tiempo promedio de espera para tomar cama en el área de recuperación, la cual cuenta con 13 camas, es de 0.00206 horas, un valor bastante aceptable. Para llevar a cabo el análisis de los escenarios, se utilizó la herramienta "Analizador de procesos" (Process Analyzer) de Arena. Se definieron 4 escenarios, la variable de control de los cuatro escenarios es el número de camas en observación. Las variables de respuesta fueron: 1) tiempo promedio para tomar cama en revisión, 2) tiempo promedio para tomar lugar en revisión, 3) \% de utilización del recurso de camas de observación y 4) tiempo promedio de espera total. Los resultados de los experimentos se presentarán en la sección de resultados y discusión.

Puede observarse que al incorporar dos camas más en el área de observación, el tiempo de espera promedio para tomar cama en observación baja a 0.1444 horas, equivalente a 8.48 minutos, todavía un valor alto; pero si en vez de 2 se incorporaran

Tabla 1: Comparativo general de puntos clave del sistema

\begin{tabular}{lccc}
\hline \multicolumn{1}{c}{ DESCRIPCIÓN } & Modelo & Real $^{1}$ & $\begin{array}{c}\% \\
\text { de diferencia }\end{array}$ \\
\hline $\begin{array}{l}\text { Promedio de pacientes que llegan en un mes } \\
\text { Tiempo de estancia promedio de los pacientes }\end{array}$ & 1815 & 1860 & $2.37 \%$ \\
$\begin{array}{l}\text { (Horas) } \\
\begin{array}{l}\text { Tiempo de espera para ser revisada y tomar } \\
\text { cama en observación (Horas) }\end{array}\end{array}$ & 0.65071 & 0.67866 & $-6.24 \%$ \\
$\begin{array}{l}\text { \% de pacientes que se revisan y se van a su } \\
\text { casa }\end{array}$ & $55.03 \%$ & $55.00 \%$ & $-0.03 \%$ \\
$\begin{array}{l}\% \text { de pacientes que se internan } \\
\text { \% de procedimientos ambulatorios }\end{array}$ & $44.97 \%$ & $45.00 \%$ & $0.03 \%$ \\
$\%$ de procedimientos normal & $15.95 \%$ & $16.20 \%$ & $0.25 \%$ \\
\hline
\end{tabular}

${ }^{1}$ Fuente: Registros y datos observados en el área de Obstetricia-Ginecología del departamento de emergencias de un hospital público de la región. 
Tabla 2: Comparativo de procedimientos

\begin{tabular}{|c|c|c|c|c|c|c|}
\hline \multirow[b]{2}{*}{ Procedimiento } & \multicolumn{3}{|c|}{$\begin{array}{l}\% \text { de ocurrencia de } \\
\text { procedimientos }\end{array}$} & \multicolumn{3}{|c|}{ Promedios de tiempos (horas) } \\
\hline & Modelo & Real $^{2}$ & Diferencia & Modelo & Real $^{2}$ & $\begin{array}{c}\% \text { de } \\
\text { diferencia }\end{array}$ \\
\hline N. ${ }^{\circ} 01$ Salpingo & $9.25 \%$ & $9.42 \%$ & $0.18 \%$ & 0.8893 & 0.9050 & $1.74 \%$ \\
\hline N. ${ }^{\circ} 02$ Biopsia Cérvix & $3.68 \%$ & $3.72 \%$ & $0.05 \%$ & 0.5003 & 0.5000 & $-0.06 \%$ \\
\hline N. ${ }^{\circ} 03$ Electrofulguración & $1.24 \%$ & $1.27 \%$ & $0.03 \%$ & 1.0002 & 1.0000 & $-0.02 \%$ \\
\hline N. ${ }^{\circ} 04$ Electrocirugía & $0.86 \%$ & $0.83 \%$ & $-0.03 \%$ & 0.5001 & 0.5000 & $-0.02 \%$ \\
\hline N. ${ }^{\circ} 05$ Crioterapia & $0.62 \%$ & $0.64 \%$ & $0.02 \%$ & 0.3340 & 0.3333 & $-0.20 \%$ \\
\hline N. ${ }^{\circ} 06$ Biopsia Endometrio & $0.30 \%$ & $0.30 \%$ & $0.01 \%$ & 1.0007 & 1.0000 & $-0.07 \%$ \\
\hline N. ${ }^{\circ} 07$ Biopsia Mama & $0.01 \%$ & $0.01 \%$ & $0.00 \%$ & 0.9500 & 0.9500 & $0.00 \%$ \\
\hline N. ${ }^{\circ} 21$ Parto Normal & $56.21 \%$ & $55.81 \%$ & $-0.40 \%$ & 0.8757 & 0.8750 & $-0.08 \%$ \\
\hline N. ${ }^{\circ} 22$ Cesárea & $10.42 \%$ & $10.47 \%$ & $0.05 \%$ & 1.2161 & 1.2650 & $3.87 \%$ \\
\hline N. ${ }^{\circ} 23$ Cesárea y Salpingo & $5.81 \%$ & $5.81 \%$ & $0.01 \%$ & 1.2193 & 1.2650 & $3.62 \%$ \\
\hline N. ${ }^{\circ} 24$ Cesárea e Histerectomía & $1.11 \%$ & $1.14 \%$ & $0.04 \%$ & 1.2526 & 1.2650 & $0.98 \%$ \\
\hline N. ${ }^{\circ} 25$ Legrado & $8.99 \%$ & $9.07 \%$ & $0.08 \%$ & 0.6785 & 0.6917 & $1.90 \%$ \\
\hline N. ${ }^{\circ} 26$ Laparotomía Exploratoria & $1.16 \%$ & $1.14 \%$ & $-0.01 \%$ & 1.5753 & 1.5750 & $-0.02 \%$ \\
\hline N. ${ }^{\circ} 27$ Colpoperineoplastía & $0.36 \%$ & $0.35 \%$ & $-0.01 \%$ & 1.5752 & 1.5750 & $-0.02 \%$ \\
\hline SUMA & $100 \%$ & $100 \%$ & & & & \\
\hline
\end{tabular}

Fuente: Registros y datos observados en el área de obstetricia-ginecología del departamento de emergencias de un hospital público de la región.

Tabla 3: Tiempos promedios de espera y número de personas esperando en filas

\begin{tabular}{lcccccc}
\hline \multirow{2}{*}{\multicolumn{1}{c}{ Filas de espera }} & \multicolumn{3}{c}{ Tiempo de espera } & \multicolumn{2}{c}{ Número de personas esperando } \\
\cline { 2 - 6 } & Promedio & Mínimo & Máximo & Promedio & Mínimo & Máximo \\
\hline Tomar cama en observación & 0.50062 & 0 & 10.5632 & 0.43758 & 0 & 12 \\
Tomar lugar en revisión & 0.10517 & 0 & 3.5522 & 0.20437 & 0 & 14 \\
Indicaciones finales & 0.02497 & 0 & 0.9232 & 0.02670 & 0 & 2 \\
Revisión & 0.02385 & 0 & 0.9759 & 0.04634 & 0 & 2 \\
Tomar personal en sala & 0.01090 & - & - & 0.01090 & - & - \\
Tomar cama de recuperación & 0.00206 & 0 & 3.0601 & 0.00180 & 0 & 6 \\
Tomar sala & 0.00161 & - & - & 0.00161 & - & - \\
Información & 0.00052 & 0 & 0.0545 & 0.00126 & 0 & 3 \\
Mostrador & 0.00046 & 0 & 0.0403 & 0.00040 & 0 & 2 \\
Tomar baño & 0.00030 & 0 & 0.1093 & 0.00059 & 0 & 1 \\
Cajero & 0.00007 & 0 & 0.0260 & 0.00001 & 0 & 1 \\
SUMA & $\mathbf{0 . 6 7 0 6 6}$ & & & & & \\
\hline
\end{tabular}

Fuente: Datos generados por el modelo validado. Actualmente, se tienen 7 camas en el área de observación pero en base a los tiempos de espera altos, estas no son suficientes con la demanda actual de los pacientes y los tiempos de estancias en observación. 


\section{RESULTADOS Y DISCUSIÓN}

La tabla 4 presenta los resultados de la evaluación de los 4 escenarios.

TABLA 4: Resultados de la evaluación de escenarios

\begin{tabular}{lcccccc}
\hline & Variable de control & \multicolumn{4}{c}{ Variables de respuesta } \\
\hline Escenario & $\begin{array}{c}\text { Número } \\
\text { de } \\
\text { réplicas }\end{array}$ & $\begin{array}{c}\text { Número de } \\
\text { camas en } \\
\text { observación }\end{array}$ & $\begin{array}{c}\text { Tiempo } \\
\text { promedio para } \\
\text { tomar cama en } \\
\text { observación }\end{array}$ & $\begin{array}{c}\text { Tiempo } \\
\text { promedio } \\
\text { para tomar } \\
\text { lugar en } \\
\text { revisión }\end{array}$ & $\begin{array}{c}\text { \% de } \\
\text { utilización } \\
\text { de camas de } \\
\text { observación }\end{array}$ & $\begin{array}{c}\text { Tiempo } \\
\text { promedio } \\
\text { de espera } \\
\text { total }\end{array}$ \\
\hline 1 (original) & 10 & 7 & 0.5006 & 0.1052 & $73.94 \%$ & 0.6688 \\
2 & 10 & 9 & 0.1444 & 0.0938 & $50.82 \%$ & 0.3056 \\
3 & 10 & 11 & 0.0348 & 0.0869 & $38.82 \%$ & 0.1912 \\
4 & 10 & 13 & 0.0060 & 0.0851 & $31.96 \%$ & 0.1584 \\
\hline
\end{tabular}

Fuente: Datos generados por el modelo validado.

4 camas adicionales, el valor baja a 0.0348 horas, equivalente a 2.09 minutos, un valor aceptable con una utilización del recurso del $38.82 \%$. Finalmente, al incorporar 6 camas más, el valor baja hasta 0.0060 horas, equivalente a 0.36 minutos, un valor bastante significativamente reducido con respecto al valor actual.

Obsérvese que al incrementar el número de camas en observación también produce una reducción del tiempo de espera para tomar lugar en revisión. Con 7 camas el tiempo de espera promedio por lugar de revisión es de 0.1052 horas (6.3 minutos) y con 13 camas se reduce a 0.0851 horas (5.11 minutos).

Aún cuando la utilización del recurso de camas solo se utilizaría en un $32 \%$, aproximadamente, se recomienda incorporar 6 camas adicionales en el área de observación, debido a que es un área de emergencia. La última columna muestra cómo se reduce el tiempo de espera total de 0.6688 horas a 0.1584 horas con 6 camas de observación adicionales. Esto resultaría en una reducción del tiempo de espera total en $76 \%$.

\section{CONCLUSIONES}

Se construyó un modelo de simulación sobre la atención de pacientes en el área de obstetriciaginecología del departamento de emergencias de un hospital de la región. El modelo de simulación permitió identificar el mayor cuello de botella del flujo de pacientes resultando ser la espera por cama en observación. En base a lo anterior, se llevaron a cabo experimentos modificando la variable del número de camas en observación y se observó el impacto en las variables de respuesta entre estas, el tiempo promedio de espera total y el porcentaje de utilización de camas en observación. En base a los resultados, se recomienda incorporar camas adicionales en el área de observación para obtener una reducción significativa en el tiempo promedio de espera total. Para realizar la mejora se requiere hacer una redistribución del área para optimizar los espacios y permitir la incorporación de las camas adicionales.

Se recomienda que se explote el potencial del modelo para realizar más análisis con otras variables como es el caso del número de personal de enfermeras, doctores e intendencia. Como trabajo futuro, también se recomienda estudiar la planeación de los procedimientos en las salas de expulsión y quirófanos.

\section{RECONOCIMIENTOS}

Este proyecto fue financiado por la Facultad de Ingeniería de la Universidad Autónoma de Baja California. Un reconocimiento especial para el personal del hospital que hizo posible la realización de este estudio. 


\section{REFERENCIAS BIBLIOGRÁFICAS}

1. Akkerman, R, y Knip M, (2004). Reallocation of beds to reduce waiting time for cardiac surgery. Healthcare management science, 7(2): 129126.

2. Clague, JE. et al. (1997). Improving outpatient clinic efficiency using computer simulation. International Journal of Healthcare Quality Assurance, 10: 197-201.

3. Denton, B. et al. (2007). Optimization of surgery sequencing and scheduling decisions under uncertainty. Healthcare management science, 10(1): 13-24.
4. Guasch, A. et al. (2005). Modelado y simulación: aplicación a procesos logísticos de fabricación y

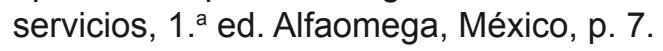

5. Kelton, D. (2004). Simulation with arena, $3{ }^{\text {th }}$ ed., McGraw-Hill, pp. 7, 261-262, 304-308.

6. Lapierre, D. y Ruiz, A. (2007). Scheduling logistic activities to improve hospital supply systems. Computers and operations research. 34(3): 624641.

7. Law, M. y Kelton, D. (2000). Simulation modeling and analysis. $3 .^{\text {th }}$ ed., McGraw-Hill, p. 390.

8. Taha, H. (1995). Investigación de operaciones. $3 .^{\text {th }}$ ed., Alfaomega, p. 11. 